\title{
Anti-Fas (CD95/Apo-I) Autoantibodies and Soluble Fas Levels Concur in T Cell Depletion in HIV Type 1 Infection
}

\author{
FRANCO SILVESTRIS, ${ }^{1}$ DANIELA GRINELLO,${ }^{1}$ ANNALISA DEL PRETE,${ }^{1}$ PAOLA CAFFORIO,${ }^{1}$ \\ MICHELE QUARTO, ${ }^{2}$ and FRANCO DAMMACCO ${ }^{1}$
}

\begin{abstract}
Deregulation of the Fas/FasL pathway in activated $T$ cells is suspected to contribute to the abnormal apoptosis that drives their progressive depletion during HIV-1 infection. However, the role of serum soluble Fas (sFas) is unclear. Here we investigated both sFas and anti-Fas IgG levels in a cohort of 227 HIV-1-infected patients with respect to their $\mathbf{T}$ cell apoptosis. By using optimized ELISAs, we found that serum titers of sFas and anti-Fas were linearly correlated in 17 severely lymphopenic subjects as compared with other patients grouped in relation to their single expression of anti-Fas and sFas, or with double-negative control patients. Cytofluorimetric measurement of the subdiploid DNA-containing cell population by both PI and TUNEL revealed an increased occurrence of cell death in vitro, in particular in patients with elevations of sFas. We also found that fresh $\mathrm{CD4}^{+}$cells from these patients showed high levels of both caspase 3 (CPP32) and its molecular targets, namely PARP and CK18. In addition, their in vitro proliferative rate was inhibited by sFas, in particular in patients with undetectable levels of the soluble receptor in vivo as well as in normal donors. In these subjects the Fas-related caspase 8 (FLICE) was significantly increased in cells treated with the recombinant Fas. These results support the contention that functionally exhausted $T$ cells may undergo apoptosis in response to the persistent in vivo stimulation by sFas. This may elucidate the described occurrence of enhanced cell death in advanced HIV-1 infection in association with serum elevations of the soluble receptor.
\end{abstract}

\section{INTRODUCTION}

D EREGULATED APOPTOSIS is considered a major pathogenetic event leading to severe $\mathrm{CD} 4{ }^{+}$lymphopenia during the human immunodeficiency virus type 1 (HIV-1) infection. ${ }^{1}$ Investigation has also shown that the tendency of $\mathrm{T}$ cells to undergo activation-induced cell death increases as infection progresses? Fas (CD95/Apo-I), a 45-kDa cell receptor involved in triggering death signals in these cells, and its cellular pathway ${ }^{3}$ play a primary role in the accelerated T cell apoptosis in HIV-1-infected patients. Both expression and sensitivity of Fas to either agonist $t^{4}$ or inert ligands ${ }^{5}$ are persistently upregulated in advanced infection stages in concurrence with the enhanced apoptosis.

Additional factors contribute to the enhanced function of the Fas pathway. The natural ligand of Fas (FasL), a type II membrane protein, is overexpressed by virus-activated lymphocytes and monocytes during the primary infection and primes autocrine suicide in $\mathrm{T}$ cells after their T cell receptor (TCR) stimulation. ${ }^{6}$ Moreover, FasL is solubilized by chronically activated $\mathrm{T}$ cells and by HIV-1-infected macrophages and may promote further triggering of the membrane-bound Fas in activated cycling T lymphocytes. ${ }^{7}$ Molecular cross-linking of Fas by agonist serum anti-Fas IgG, as reported by ourselves ${ }^{8}$ and others, ${ }^{9}$ also results in activation of the transducing death signal pathway involving the sequential cleavage of caspases, a family of aspartate-specific cysteine proteases that propagate the biochemical events responsible for apoptosis. ${ }^{10}$

Accumulating evidence also suggests that serum levels of soluble Fas (sFas) may increase during infection, ${ }^{9,11}$ even in concomitance with enhanced $\mathrm{T}$ cell apoptosis, ${ }^{12}$ thus attributing a potential role to its solubilization. This mechanism of membrane receptor shedding by activated cells is suspected to play a role in the fine regulation of the immune response. Serum

\footnotetext{
${ }^{1}$ Department of Biomedical Sciences and Human Oncology, Section of Internal Medicine and Clinical Oncology, University of Bari, 70124 Bari, Italy.

${ }^{2}$ Institute of Hygiene and Preventive Medicine, University of Bari, 70124 Bari, Italy.
} 
elevations of some T cell receptors, including HLA class I and II molecules, have been detected in allografts ${ }^{13}$ and healthy individuals. ${ }^{14}$ In addition, increased levels of TCR chains as well as members of the TNF receptor family, namely both type I (p55) and II (p75) receptors, Fas, and FasL, have been related to the persistent $\mathrm{T}$ cell activation that occurs in chronic inflammatory and autoimmune diseases, ${ }^{15,16}$ and in HIV-1 infection. ${ }^{17,18}$

The function of soluble receptors is at present under intensive investigation. It has been reported that sHLA class I molecules induce a FasL-mediated apoptosis in cytotoxic T lymphocytes (CTLs), ${ }^{19}$ and this suggests a downregulatory effect on the immune response. On the other hand, the secretion of sFas by activated T cells was postulated to prevent their own apoptosis. This putative role of apoptosis blocker was attributed to a few isoforms of the receptor lacking the transmembrane domain, ${ }^{20}$ that were shown to inhibit the FasL of CD8 ${ }^{+}$ cells in vitro, thus explaining the defect of suppressor function during autoimmunity. ${ }^{21}$ Elevated levels of sFas also block FasL in patients with leukemias, ${ }^{22}$ whereas a decrease of this hypothetical blocker accelerates the apoptosis of $\mathrm{T}$ cells in HIV-1 infection. ${ }^{12,18}$ However, both Fas cellular overexpression and release occur predominantly in advanced stages in coincidence with the highest expression of apoptosis, ${ }^{23}$ in agreement with the hypothesis that the increment of sFas influences rather than prevents peripheral cell death.

The present study was addressed to explore the significance of sFas during HIV-1 infection in relation to increased apoptosis. By using multiple analyses in a cohort of patients at different clinical stages, we found that serum levels of sFas paralleled the extent of peripheral $\mathrm{T}$ cell apoptosis. Interestingly, major elevations were detectable in patients showing circulating anti-Fas antibodies, in keeping with enhanced cell death.

\section{MATERIALS AND METHODS}

\section{Patients and serological screening tests}

The study was carried out in 227 HIV-1-infected individuals with different clinical features according to the Centers for Disease Control (CDC, Atlanta, GA). ${ }^{24}$ Sera were collected from subjects periodically examined during their treatment at the outpatient clinic of the Department of Internal Medicine and Oncology and from patients included in the Program of AIDS Prevention at the Department of Hygiene and Preventive Medicine of the University of Bari (Bari, Italy). Patients with advanced disease were also recruited from the Infectious Diseases Division of this university. The majority of patients with previous or coexisting lymphopenia were included in antiretroviral treatment protocols and several of those with advanced HIV1 disease were receiving the triple-combination highly active antiretroviral therapy (HAART). All subjects gave their informed consent, and the study was approved by the ethical committee of the University of Bari. Sera were divided into aliquots and stored at $-80^{\circ} \mathrm{C}$ until use. Patients with positive values of anti-Fas antibodies and/or sFas at the screening assays were recalled and asked to provide a further blood sample for immunologic tests, which included a peripheral CD4 ${ }^{+}$cell count and measurement of peripheral $\mathrm{T}$ cell apoptosis.
Differential enzyme-linked immunosorbent assays (ELISAs) were used as previously reported to detect the levels of both anti-Fas ${ }^{8}$ molecules and sFas. ${ }^{25}$ Briefly, the 96 -well plates were separately incubated with the chimeric recombinant (r) Fas-Fc (Immunotech, Marseille, France) as antigen, or with the CH11 anti-Fas (IgM) monoclonal antibody (MAb) (Immunotech) in carbonate buffer, $\mathrm{pH}$ 9.6, and further supplemented with sera at 1:10 dilution in phosphate-buffered saline (PBS) containing fetal calf serum (FCS) and dry milk. After $5 \mathrm{hr}$ of incubation, the plates coated with $\mathrm{rFas}$ were washed and supplemented with equivalent amounts of mouse IgG1 MAbs to human $\kappa$ and $\lambda$ chains (Immunotech). The test was then completed by a peroxidase-conjugated goat anti-mouse IgG to detect Fas-reactive IgG and developed with $o$-phenylenediamine (OPD). Conversely, sFas was evaluated with a second anti-Fas MAb, namely IgG1 from clone ZB4 (Immunotech), in our sandwich assay, which was optimized to provide high sensitivity. The test was completed by a standard procedure using the labeled goat anti-mouse IgG and OPD addition. Each test was performed in triplicate and positive significance was attributed to values of absorbance higher than the mean +3 SD for the entire HIV$1^{+}$population. Soluble Fas levels were also measured in positive sera by a commercial kit (Alexis It., Vinci, Florence, Italy) and compared with data from our own assay.

\section{Peripheral $T$ cell phenotype analysis}

Patients with serum positivities to Fas, and/or elevations of sFas, provided their blood samples for cellular studies. Peripheral T lymphocytes were prepared by Ficoll-Hypaque centrifugation, removal of adherent cells, incubation with sheep red cells, and subsequent gradient centrifugation to discharge nonE-rosetting cells. Phenotyping was performed by flow cytometry and the $\mathrm{CD}^{+}$cell count was completed in a FACScan (Becton Dickinson, Mountain View, CA). In addition, CD4 ${ }^{+}$ cell preparations were obtained with an MACS CD4 ${ }^{+} \mathrm{T}$ cell isolation kit (Miltenyi Biotec, Auburn, CA), which provided $>90 \%$ cell enrichment, and then used for functional assays. Double-fluorescence analysis using the phycoerythrin (PE)conjugated MAb to Fas from clone UB2 (Immunotech) in combination with the fluorescein isothiocyanate (FITC)-conjugated MAb to CD4 molecule was also adopted to measure the receptor expression on peripheral cells.

\section{Measurement of apoptosis}

The spontaneous apoptosis of freshly derived T cells was initially evaluated by cytofluorimetric measurement of cell populations with subdiploid DNA peak by propidium iodide (PI), as described. ${ }^{5}$ Aliquots of cell preparations were also examined in parallel with the Apo2.7 MAb to a 38-kDa mitochondrial protein regarded as an early apoptosis marker, since viable cells are negative or weakly positive. ${ }^{26}$ Its expression was revealed by flow cytometry, using the permeabilizing protocol with digitonin and the PE-conjugated Apo2.7 (Immunotech). A further approach to measure the extent of apoptosis in fresh $\mathrm{T}$ cells included the terminal deoxynucleotidyltransferase (TdT)-mediated dUTP nick end labeling (TUNEL) technique, which uses fluorescein-dUTP to label the DNA strand breaks during apoptosis. This assay was completed by flow cytometry according to the manufacturer instructions (Boehringer-Mannheim, Mi- 
lan, Italy). Each evaluation was performed in triplicate and in parallel with fresh $\mathrm{T}$ cell preparations from 16 uninfected healthy donors.

\section{Evaluation of caspase activation}

Further experiments were performed to measure both the expression and activation of a few caspases in isolated $\mathrm{CD} 4{ }^{+}$cells from both patients and controls. We used fluorescence analysis to measure the constitutive expression of caspase 3 (CPP32) (rabbit antiserum; Alexis It.) and its activation by detection of both the $12-\mathrm{kDa}$ cleaved subunit, using a specific MAb (PharMingen, San Diego, CA), and the proteolytic cleavage of poly(ADP)ribose polymerase (PARP) by the appropriate MAb (New England BioLabs, Hitchin, UK). Although several of
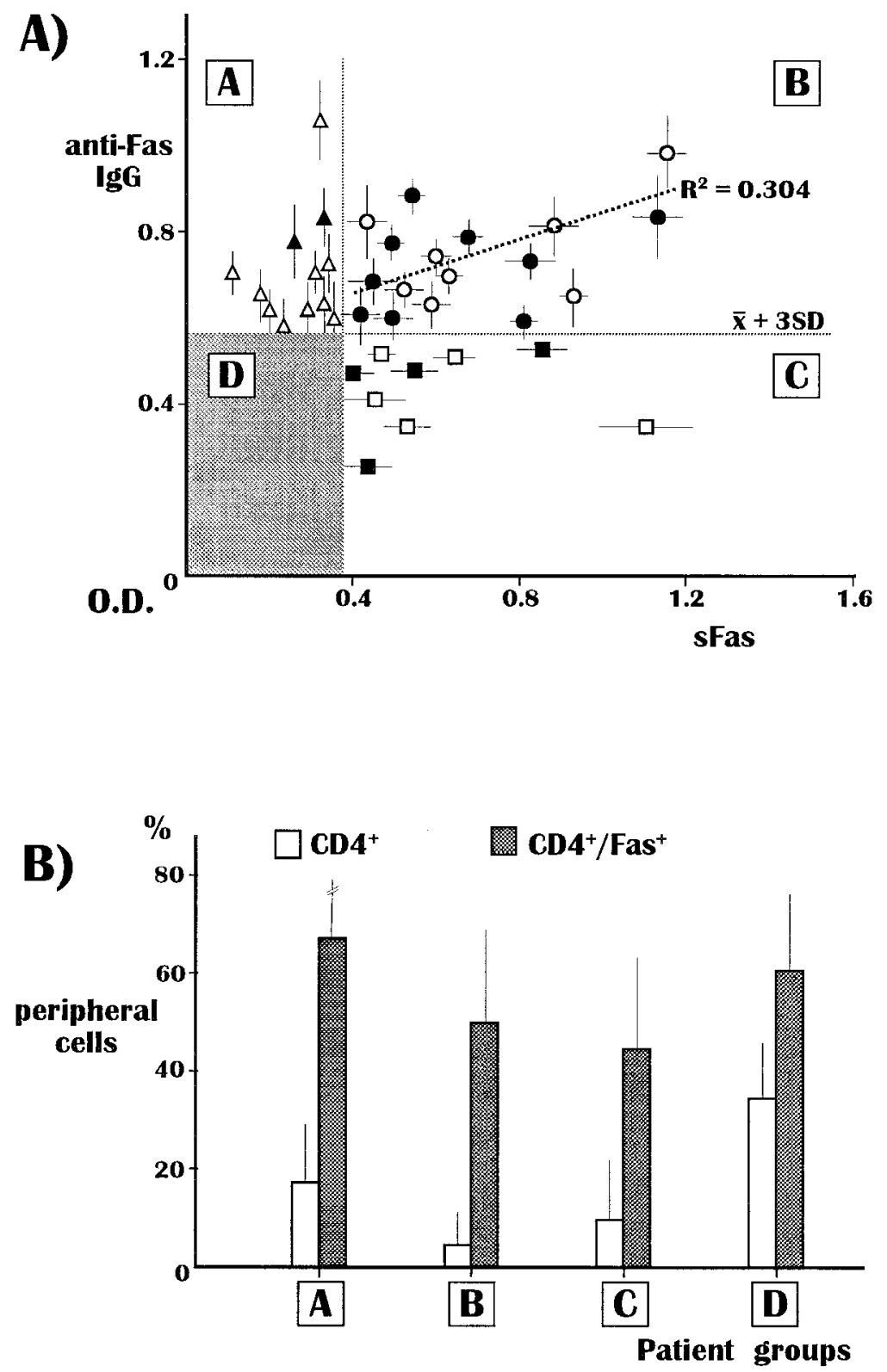

FIG. 1. (A) Correlative analysis of ELISA values of IgG to Fas with the soluble isoform of Fas detected in sera from 227 HIV1 -infected individuals at different CDC stages. Levels of absorbance higher than the mean +3 SD from the entire HIV-1 population were considered positive for each parameter. This evaluation identified four groups of patients in relation to positive or negative values. The double-positive group of patients (group B) showed a linear correlation between both parameters $\left(R^{2}=\right.$ 0.304 ) and included a higher number of subjects with AIDS (solid symbols) with respect to other groups. (B) Mean values of percent expression of both $\mathrm{CD}^{+}{ }^{+}$and $\mathrm{CD}^{+} / \mathrm{Fas}^{+}$cell subsets in four groups of HIV-1-infected individuals, divided in relation to their serum positivities as anti-Fas antibodies and soluble Fas. Extreme levels of lymphopenia were observed in subjects showing serum elevations of both parameters (group B) with respect to the moderate depletion of CD4 ${ }^{+}$cells observed in doublenegative patients (group D). Mean values of this analysis were significantly different $(p<0.02)$. However, Fas was uniformly overexpressed by these cells, although with a slight intergroup variability. 
these reagents had been optimized by their manufacturers for immunoblotting methods and used by ourselves in previous work, ${ }^{27}$ we also tested their reliability in flow cytometry assay. ${ }^{28}$ Briefly, $\mathrm{CD} 4{ }^{+}$cells were fixed and then permeabilized and separately treated with each MAb. Last, the assay was completed by use of the FITC-conjugated antibody prior to flow cytometric analysis. As negative controls we used a nonspecific MAb with the secondary FITC-conjugated antibody.

Cleaved cytokeratin 18 (CK18) was also investigated as an additional product of caspase activation. ${ }^{29}$ Thus, the cells were fixed in ice-cold methanol, incubated with the FITC-conjugated MAb to cleaved CK18 (Boehringer-Mannheim), and then analyzed by the FACScan.

\section{$\mathrm{CD}^{+}$cell stimulation with sFas and functional analysis}

A number of experiments were also devoted to exploring the effect of recombinant sFas on fresh $\mathrm{T}$ cells in vitro. Thus, $0.5 \times$ $10^{6}$ isolated CD4 ${ }^{+}$cells from several patients of each group and from 15 normal donors were incubated overnight and for $24 \mathrm{hr}$ in complete medium supplemented with increasing amounts of sFas $(0.01-10 \mu \mathrm{g} / \mathrm{ml})$. The cells were then washed and used in functional tests. We first explored their proliferation rate after sFas stimulation by measuring the $\left[{ }^{3} \mathrm{H}\right]$ thymidine incorporation. Briefly, cell cultures included the $\left[{ }^{3} \mathrm{H}\right]$ thymidine at $7400 \mathrm{~Bq} / \mathrm{well}$ and the proliferation was assessed with a $\beta$ counter (Beckman, Palo Alto, CA) as described. ${ }^{5}$ Parallel cell suspensions were also measured in the FACScan for the expression of both PARP and CK18, and caspase 8 (FLICE), by relative MAbs (MAb to FLICE: Biosource International, Camarillo, CA). These evaluations included proper controls of parallel unstimulated cell suspensions. In addition, further controls were prepared by culturing the cells with sFas preincubated for 90 min with the mouse IgG1 anti-Fas MAb from clone ZB4 (Immunotech) at $10 \mu \mathrm{g} / \mathrm{ml}$, as an attempt to neutralize the soluble receptor.

\section{Statistical analysis}

Differences between means of groups of data were calculated by using the Student $t$ test. ANOVAR followed by a Stu- dentized range test was used for comparing the proportions of cell subsets whereas the correlation of optical density (OD) data was assessed by linear regression analysis.

\section{RESULTS}

\section{Occurrence and distribution of anti-Fas antibodies and sFas}

Figure 1A shows the distribution of serum levels of both antiFas IgG and sFas in the whole HIV-1-infected population. We identified different groups of patients in relation to the positivity for each serological marker, whose significance was attributed to values higher than the mean $+3 \mathrm{SD}$ of absorbance from the entire HIV-1 population. Therefore, this was divided into four groups: (A) 12 patients positive to anti-Fas IgG detection; (B) 17 with both anti-Fas and sFas positivities; (C) 9 with significant serum elevations of sFas; and (D) a double-negative group that included 189 subjects. We also reevaluated with a commercial kit the levels of $\mathrm{sFas}$ in positive sera from both groups B and C, and found results comparable with our assay. ${ }^{25}$ Therefore the distribution in groups defined an apparent association between both positivities, in particular in group B, whose sera showed a linear correlation of their values $\left(R^{2}=\right.$ $0.304)$.

The expression of serum anti-Fas molecules and/or sFas was independent of the CDC disease stage, since patients at CDC stage IV with AIDS (Fig. 1A, solid symbols) were present in all groups, although they occurred predominantly in the double-positive population. However, the incidence of each marker within the entire group of patients was in agreement with previous studies by ourselves ${ }^{8,30}$ and others. ${ }^{9}$ Figure 1B compares the levels of both $\mathrm{CD}^{+}{ }^{+}$and $\mathrm{CD} 4{ }^{+} / \mathrm{Fas}^{+}$cells between these groups. As shown, an apparent discrepancy within each cell population was recorded. Although groups $\mathrm{A}, \mathrm{B}$, and $\mathrm{C}$ were composed of few patients, we found significantly decreased values of the $\mathrm{CD}^{+}$cell population in each group as compared with the double-negative patients in group $\mathrm{D}(p<0.02$ in all instances). However, group B showed the lowest mean value $\left(\mathrm{CD} 4^{+}\right.$cells $\left.=6.8 \pm 5.2 \%\right)$, an effect of the higher number of

Table 1. Peripheral T Cell Apoptosis, Measured as Extent of Subdiploid DNA-Containing Cells, in Groups of Patients in Relation to the Occurrence of Severe Lymphopenia ${ }^{\text {a }}$ With AIDS

\begin{tabular}{|c|c|c|}
\hline Subjects & $\begin{array}{l}\text { Peripheral T cells with } \\
\text { subdiploid DNA content }\end{array}$ & $\begin{array}{c}\text { Occurrence of } \\
\text { severe lymphopenia } \\
\text { with AIDS }\end{array}$ \\
\hline \multicolumn{3}{|l|}{ HIV-1 patients } \\
\hline Group A & $34 \pm 4$ & $2 / 12(16 \%)$ \\
\hline Group B & $41.2 \pm 5$ & $9 / 17(52 \%)$ \\
\hline Group C & $38.5 \pm 7$ & $4 / 9(44 \%)$ \\
\hline Group D & $24.8 \pm 5^{c}$ & $16 / 189(8.4 \%)$ \\
\hline $\begin{array}{l}\text { Uninfected control } \\
\text { subjects }\end{array}$ & $9.1 \pm 3.8^{\mathrm{d}}$ & \\
\hline
\end{tabular}

${ }^{\mathrm{a} C D} 4^{+}$cells $<200 / \mathrm{ml}$.

${ }^{\mathrm{b}}$ Numbers refer to the percentage of positive cells (mean \pm SD) detected by flow cytometry.

'Investigated in 21 subjects.

${ }^{\mathrm{d}}$ Investigated in 16 normal donors. 
patients with AIDS. On the other hand, we recorded a different pattern of Fas expression by these cells. The receptor was indeed overexpressed in group B as well as in other groups of patients with no intergroup differences $(p>0.5$ in all instances), although the highest mean value occurred apparently in group A. These experiments revealed that patients with significant elevations of both anti-Fas and sFas showed the lowest levels of circulating $\mathrm{CD}^{+}{ }^{+}$cells and that the apoptogen receptor was equally expressed by these cells.

\section{Double-positive patients showed enhanced $T$ cell apoptosis}

The $\mathrm{T}$ cell apoptosis of patients from groups A, B, and C, and 21 from group $\mathrm{D}$, was evaluated by flow cytometry of the subdiploid DNA-containing cell subset within the peripheral fresh $\mathrm{T}$ lymphocytes after PI staining. Table 1 illustrates the mean level of this population within each group with respect to the incidence of the severe lymphopenia that defines AIDS or CDC stage IV $\left(\right.$ CD4 $^{+}$cells $\left.<200 / \mu 1\right)$. In spite of the different numbers of patients, we observed an expansion of the apoptotic population as a mean value of the relative patient subset, in particular in group B $(41.2 \pm 5 \%)$. By contrast, a decreased value was revealed in group D $(24.8 \pm 5 \%)$, although to a higher magnitude than in the uninfected control group $(9.1 \pm 3.8 \%)$. A general pattern of abnormal apoptosis was also detected in subjects from groups $\mathrm{A}$ and $\mathrm{C}$ as compared with the controls, thus confirming its substantial deregulation during the clinical course of HIV-1 infection. In addition, the variable levels of peripheral $\mathrm{T}$ cell apoptosis were in apparent parallelism with the distribution of severely lymphopenic patients. Notably, these patients were predominantly in group B $(52 \%)$, in association with the highest extent of peripheral $\mathrm{T}$ cell death.

To explore the effect in vivo of the persistent $\mathrm{T}$ cell stimulation by either Fas-reactive IgG or sFas during nonlymphopenic stages of the infection, a number of patients with CDC stages I to III of clinical disease from all groups were investigated by both TUNEL assay and the detection of Apo2.7-reactive antigen to determine the extent of their $\mathrm{T}$ cell apoptosis. Table 2 shows their individual values with respect to the relative mean levels measured in healthy controls. We recorded a prevalent expansion of the TUNEL-positive cell population, particularly in subjects from group B as compared with those in group D, and with controls. Mean values within each group indicated that the occurrence of TUNEL-positive cells in freshly derived lymphocytes was significantly higher in both groups B and $\mathrm{C}$ with respect to double-negative subjects, and to controls ( $p<0.01$ in all instances). By contrast, the extent of the Apo2.7-reactive population was higher in all patients than in controls $(p<0.02)$. Since this mitochondrial protein is considered an early marker of apoptosis, its prevalent expression in the majority of HIV-1-infected subjects may be associated with the enhancement of the phenomenon, during the nonlymphopenic stages of HIV-1 infection. Figure 2 illustrates a few representative patterns from two patients from groups B and D. As shown, a difference was evident when comparing the occurrence of apoptotic cells (M2 peak) in peripheral lymphocytes treated by TUNEL since it was more appreciable in patient HY-609 from group B. By contrast, the Apo2.7-reactive protein was expressed at slightly different levels (M2), sug-
Table 2. Apoptosis Parameters in 23 Nonlymphopenic Patients ${ }^{\text {a }}$ Compared with Control DATA FROM 16 UNINFECTED INDIVIDUALS ${ }^{b}$

\begin{tabular}{|c|c|c|c|}
\hline Subjects & $\begin{array}{l}C D C \\
\text { stage }\end{array}$ & TUNEL & Apo 2.7 reactive \\
\hline \multicolumn{4}{|l|}{ HIV-1 patients } \\
\hline \multicolumn{4}{|l|}{ Group A } \\
\hline Patient DI-S14 & I & 42.6 & 57.0 \\
\hline Patient DI-078 & II & 59.8 & 72.3 \\
\hline Patient HY-011 & III & 51.3 & ND \\
\hline Patient HY-304 & II & 64.0 & 63.8 \\
\hline Patient ID-CF36 & II & 40.4 & 72.3 \\
\hline Mean \pm SD & & $\overline{51.6 \pm 10.3}$ & $\overline{66.3 \pm 7.4}$ \\
\hline \multicolumn{4}{|l|}{ Group B } \\
\hline Patient DI-R19 & II & 67.0 & 58.9 \\
\hline Patient HY-432 & II & 71.4 & 78.5 \\
\hline Patient HY-609 & II & 62.5 & 84.3 \\
\hline Patient HY-903 & III & 84.1 & 87.3 \\
\hline Patient ID-BQ09 & $\mathrm{I}$ & 65.5 & 71.4 \\
\hline Patient ID-F009 & II & 59.4 & 80.0 \\
\hline Mean $\pm \mathrm{SD}$ & & $\overline{68.3 \pm 8.7}$ & $\overline{76.7 \pm 9.8}$ \\
\hline \multicolumn{4}{|l|}{ Group C } \\
\hline Patient DI-R10 & II & 53.2 & 75.2 \\
\hline Patient DI-T23 & III & 78.5 & 82.3 \\
\hline Patient HY-118 & II & 67.5 & 77.3 \\
\hline Patient HY-307 & II & 59.0 & 59.4 \\
\hline Patient ID-CT98 & I & 80.4 & 71.3 \\
\hline Mean $\pm \mathrm{SD}$ & & $\overline{67.7 \pm 11.8}$ & $\overline{73.1 \pm 8.6}$ \\
\hline \multicolumn{4}{|l|}{ Group D } \\
\hline Patient DI-F51 & II & 38.9 & 71.8 \\
\hline Patient HY-512 & I & 19.6 & 63.0 \\
\hline Patient HY-602 & II & 27.5 & 59.3 \\
\hline Patient HY-804 & III & 46.8 & ND \\
\hline Patient ID-F203 & $\mathrm{I}$ & 39.2 & 61.8 \\
\hline Patient ID-R014 & II & 21.0 & 52.2 \\
\hline Patient ID-S036 & II & 27.6 & 69.6 \\
\hline Mean $\pm \mathrm{SD}$ & & $\overline{35.7 \pm 15}$ & $\overline{62.9 \pm 7.1}$ \\
\hline \multicolumn{4}{|l|}{ Healthy controls } \\
\hline Mean $\pm S D$ & & $9.1 \pm 3$ & $22.5 \pm 12$ \\
\hline
\end{tabular}

Abbreviation: ND, Not done.

${ }^{\mathrm{a}} \mathrm{CDC}$ stage I-III.

${ }^{b}$ Numbers refer to percentages of positive cells obtained by flow cytometry. Mean values \pm SD are related to each group.

gesting the persistent susceptibility to apoptosis in these patients.

A further attempt to investigate the actual activation of apoptosis in vivo included the evaluation of caspase 3 for measurement of caspase activity in a restricted number of patients from each group, namely three from group A (patients DI-078, HY304, and ID-CF36), four from group B (patients DI-R19, HY432, HY-609, and ID-F009), three from group C (patients DIR10, HY-118, and HY-307), and four from group D (patients DI-F51, HY-602, ID-R014, and ID-S036) in relation to their apparently homogeneous disease. These subjects showed both immunologic data and clinical parameters corresponding to CDC stage II and were enrolled in a similar treatment program that included conventional antiretroviraldrugs. Thus, their fresh $\mathrm{CD}^{+}{ }^{+}$cells were evaluated by flow cytometry in their constitutive expression of caspase 3 as well as its $12-\mathrm{kDa}$ activated isoform, PARP, and the cytoskeletal protein CK18 cleaved 
TUNEL

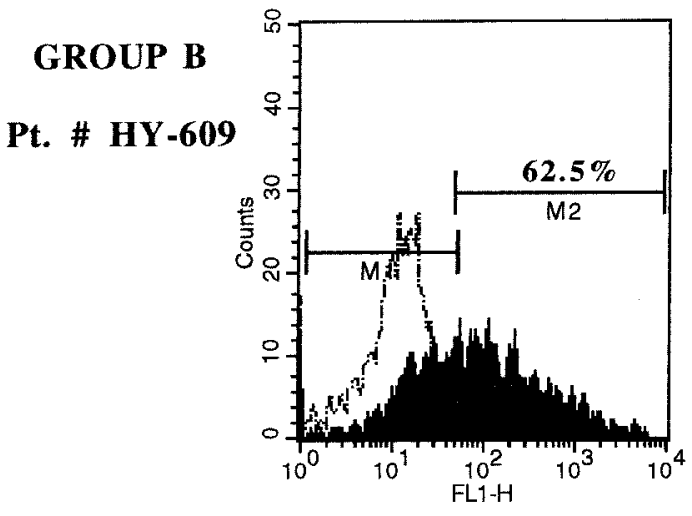

Apo2.7

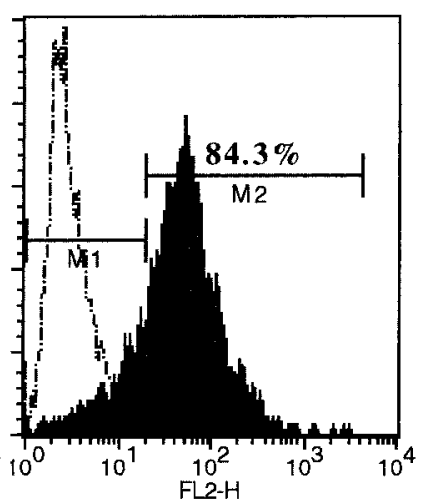

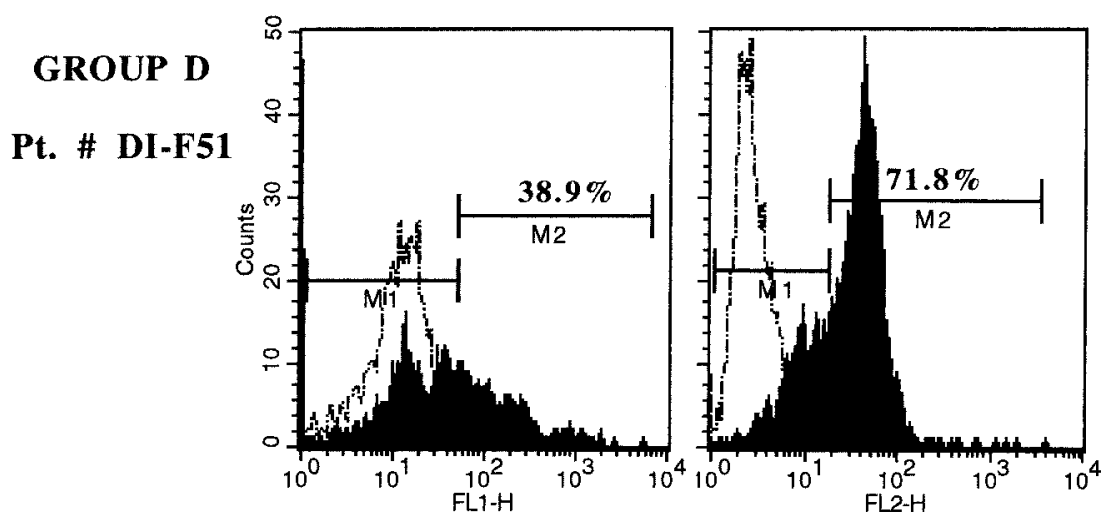

FIG. 2. Patterns of apoptosis measured in vitro in fresh T cells from two representative patients of groups B and D. The flow cytometry analysis revealed a clear-cut prevalence of cell death (M2) in cells from patients with serum elevations of sFas and/or anti-Fas, using the TUNEL method. Conversely, the Apo2.7-reactive mitochondrial antigen as early marker of apoptosis was also expressed by patients of group D. Percentages indicate the number of TUNEL- and Apo2.7-positive cells. The negative control (M1) is depicted by the broken line.

\section{CPP32}

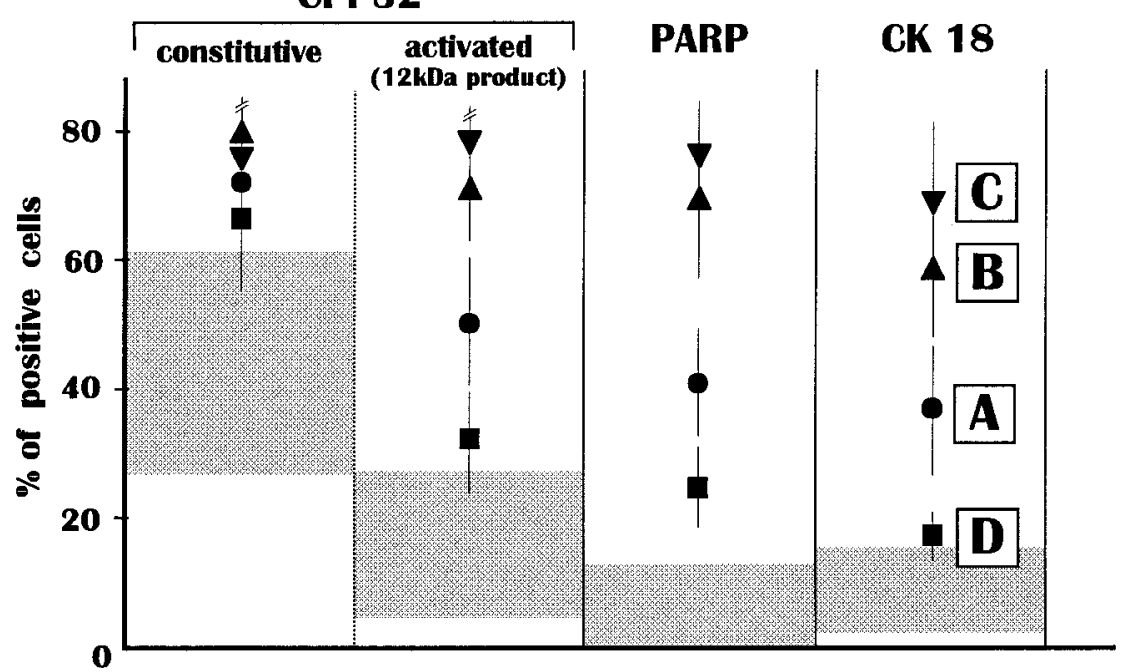

FIG. 3. Measurement of caspase expression as both native and cleaved products in freshly derived CD ${ }^{+}$cells from patients with similar clinical disease (CDC stage II). Mean values from each group are shown. Although a comparable presence of caspase 3 was detectable in $\mathrm{CD}^{+}{ }^{+}$cells from most patients, its products, including both $12-\mathrm{kDa}$ subunit and 89-kDa PARP, and the cytoskeletal cytokeratin 18 cleaved product (CK 18), were significantly expressed ( $p<0.02$ in all instances) by patients of groups $\mathrm{B}$ and C. Patients from group A showed a moderate presence in vivo of these cleavage products, whereas a slight increase was recorded in cells from patients grouped in D with respect to control normal values (shaded areas). 
within the cytoplasm by differentially activated caspases. ${ }^{29}$ The assay included the parallel measurement of caspase expression in $\mathrm{CD}^{+}$cells from the healthy controls to provide reference values. Figure 3 shows the results of this analysis. Although the constitutive expression of caspase 3 was substantial in the majority of $\mathrm{CD}^{+}$cell preparations and slightly higher than the relative control value, the persistence of activated caspases was predominant in cells from patients of groups B and C. In fact, both 12-kDa and PARP fragments were detected at significant levels in cells from these patients with respect to the other groups and to control values ( $p<0.02$ in all instances).

Similarly, an expansion of $\mathrm{CK} 18^{+}$cell population $(>60 \%)$ was observed in the same cell preparations $(p<0.01$ as compared with control values) whereas the cleaved cytoskeletal protein was moderately expressed by those from the other groups of patients. Detection of cleaved products of caspases in fresh cells suggested that the apoptotic pathway was functionally active in vivo in apparent relation to soluble potential activators, such as sFas and/or Fas-reactive IgG, rather than to the clinical stage.

\section{In vitro effects of sFas}

The next experiments were addressed to assess whether the abnormal caspase activation observed in vivo in patients from groups $\mathrm{B}$ and $\mathrm{C}$ was attributable to $\mathrm{sFas}$. Therefore, we measured the in vitro effect of the soluble receptor on caspase ex- pression in isolated $\mathrm{CD}^{+}{ }^{+}$cells from the same CDC stage II subjects receiving the antiretroviral treatment. The cell suspensions were incubated with sFas and subsequently evaluated for their proliferation rate as well as for caspase 8 expression. Figure 4 shows a dose-dependent effect of sFas measured after overnight incubation. A major inhibition $(>50 \%)$ was recorded in cells from the healthy controls after administration of a 1$\mu \mathrm{g} / \mathrm{ml}$ concentration of soluble receptor. This inhibitory effect was further enhanced by increasing its amounts within the cell cultures. However, a similar result was also observed in cell preparations from patients of group $\mathrm{D}$, in contrast to a moderate inhibition of the proliferation rate $(\sim 30 \%)$ detected in patients in group A. On the other hand, the cell cultures from patients of groups $\mathrm{B}$ and $\mathrm{C}$ whose baseline proliferation was constitutively depressed (mean value of $\left[{ }^{3} \mathrm{H}\right]$ thymidine uptake, $2015 \mathrm{cpm}$ ) were poorly affected by the increasing amounts of the soluble receptor. The suppression induced by sFas on CD4 ${ }^{+}$ cells from double-negative patients and from the control group was highly significant as compared with the other groups of patients ( $p<0.01$ in all instances). The specificity of this suppression was confirmed by treatment of the soluble receptor with the ZB4 anti-Fas MAb prior to the cell stimulation, in that the cell cultures were restored in their proliferation extent. These tests proved that a striking inhibition of cell proliferation was induced in vitro by the soluble isoform of the receptor.

We also measured the apoptogen potential of sFas in vitro by evaluating the changes in caspase 8 expression. Figure $5 \mathrm{~A}$

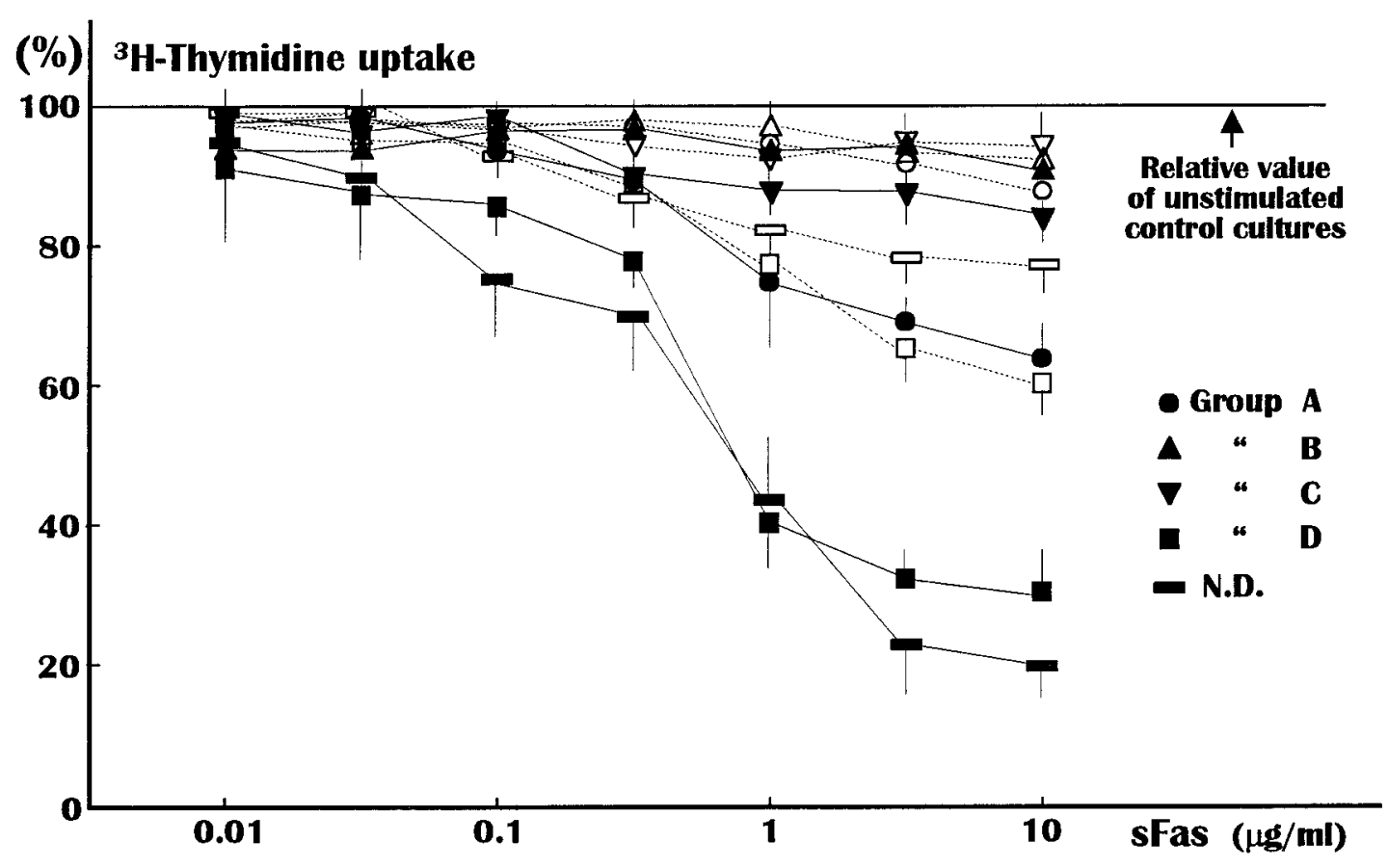

FIG. 4. Dose-dependent inhibition of $\mathrm{CD}^{+}$cell proliferation rate by sFas as a percentage of $\left[{ }^{3} \mathrm{H}\right]$ thymidine incorporation in culture. Symbols refer to patients grouped in relation to their serum detection of anti-Fas and/or sFas. Significant inhibition $(p<$ 0.01) was noted in cell preparations from double-negative patients (group D) as well as in those from control donors by the administration of a $1-\mu \mathrm{g} / \mathrm{ml}$ concentration of the soluble receptor (solid symbols). This cellular suppression was almost completely abolished by the pretreatment of sFas with a $10-\mu \mathrm{g} / \mathrm{ml}$ concentration of ZB4 IgG1 MAb anti-Fas (open symbols). Similarly, cell preparations from group A were moderately suppressed by sFas and restored in their proliferative extent by ZB4. By contrast, the basal depressed proliferation of cells $(\sim 2015 \mathrm{cpm})$ from both groups B and C was not influenced by the sFas even at higher concentrations. N.D., Normal donors. 

FLICE-positive cells

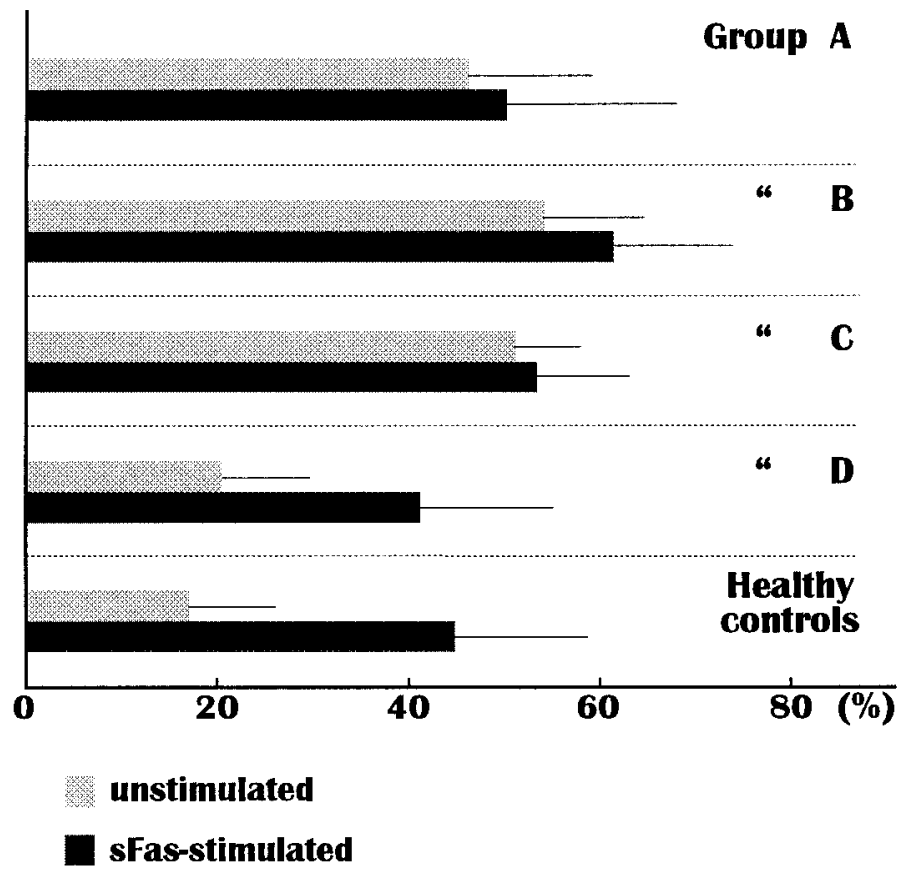

FIG. 5. (A) Changes in cellular caspase 8 (FLICE) expression by treatment with sFas. The enriched CD $4^{+}$preparations from patients included in groups $\mathrm{A}, \mathrm{B}$, and $\mathrm{C}$ expressed a basal accumulation of the caspase that was not influenced by the soluble receptor. By contrast, the percentage of positive cells in cultures from patients of group D, as well as from the control donors, namely $\mathrm{CD}^{+}$cells not preactivated in vivo by serum sFas, significantly increased in response to the treatment with the soluble receptor $(p<0.02$ in both instances). Data represent means + SD of positive cells in flow cytometry. (B) Representative cytofluorimetric patterns of variations in caspase 8 expression in individual CD4 ${ }^{+}$cell preparations from each group of patients. The maximal increase in positive cells, represented as percentages in M2 peaks, was detected in cultures from double-negative patients and from the uninfected controls. M1 peaks show the extent of negative control cells.

shows a differential pattern of this caspase both in its basal expression and in response to sFas stimulation. We recorded its peculiar accumulation in unstimulated $\mathrm{CD} 4{ }^{+}$cells from patients enrolled in groups $\mathrm{A}, \mathrm{B}$, and $\mathrm{C}$, whose levels remained mostly unchanged after the incubation with sFas $(p>0.5$ in each intragroup measurement). By contrast, a moderate expression of the caspase was detected in double-negative patients, and in the control group. The number of positive cells was at least doubled in both groups of subjects in response to the treatment with sFas, thus showing a significant increase in caspase expression with respect to the corresponding untreated cell preparations $(p<0.02)$. Interestingly, in both instances the differential value was always higher than in other groups of patients.

Figure $5 \mathrm{~B}$ shows the flow cytometry changes of caspase 8 in sFas-treated cells from a representative patient of each group. As can be seen, maximal increment of positive cells occurred within the cultures of both patient DI-F51 from group D and the normal donor F45-3 whereas small variations were observed in subjects from the other groups after treatment with the soluble receptor.

These results provided definite evidence of the role of sFas as caspase activator, since this effect was inducible in cells from subjects with no serum elevations of the soluble factor in vivo, as in normal donors.

\section{DISCUSSION}

The molecular mechanisms that induce enhanced $\mathrm{T}$ cell apoptosis in HIV-1 disease are still unclear. The abnormal response of chronically activated $\mathrm{T}$ lymphocytes to persistent antigen stimulation is regarded as the major derangement driving the progressive $\mathrm{CD}_{4}{ }^{+}$cell depletion. Here we explored the role of soluble molecules potentially correlated with this modality of active cell death.

Measurement of both anti-Fas IgG and the soluble isoform of Fas revealed a correlation of their serological elevations in a small group of patients showing severe $\mathrm{CD}^{+}{ }^{+}$cell lymphopenia, and prevalence of AIDS. Such low $\mathrm{CD}^{+}{ }^{+}$cell values were paralleled by an increase in $\mathrm{T}$ cell apoptosis detected by both TUNEL and the measurement of the mitochondrial marker reactive to Apo2.7. That Fas-reactive antibodies trigger the receptor in its membrane-bound form was proved in previous studies showing an inverse relation of their serum levels with the $\mathrm{CD} 4{ }^{+}$cell counts in groups of patients observed longitudinally by repeated measurements. ${ }^{8,9,30}$ Therefore, their detection in sera of patients from both groups A and B was in agreement with the concurrent enhanced apoptosis of their peripheral cells. However, we investigated the effect of sFas since patients with isolated serum elevations of the receptor (group 
B
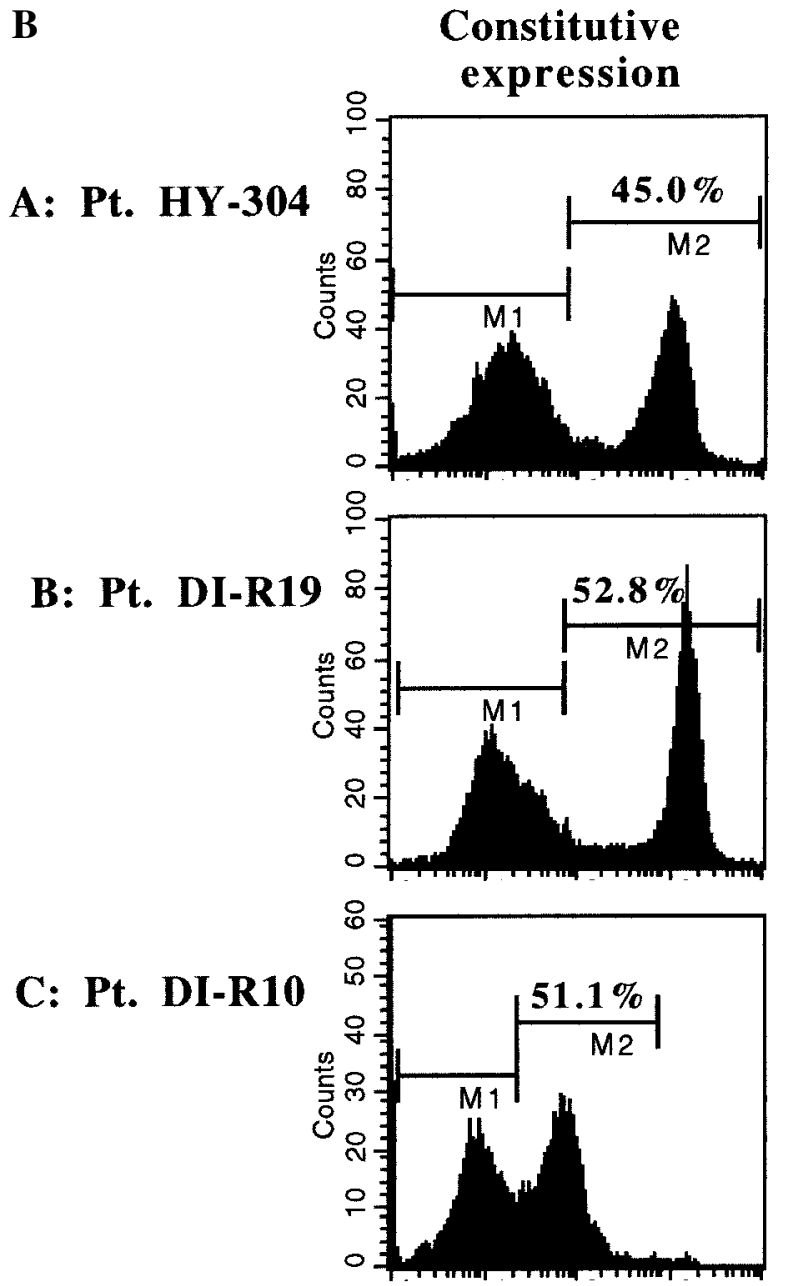

D: Pt. DI-F51
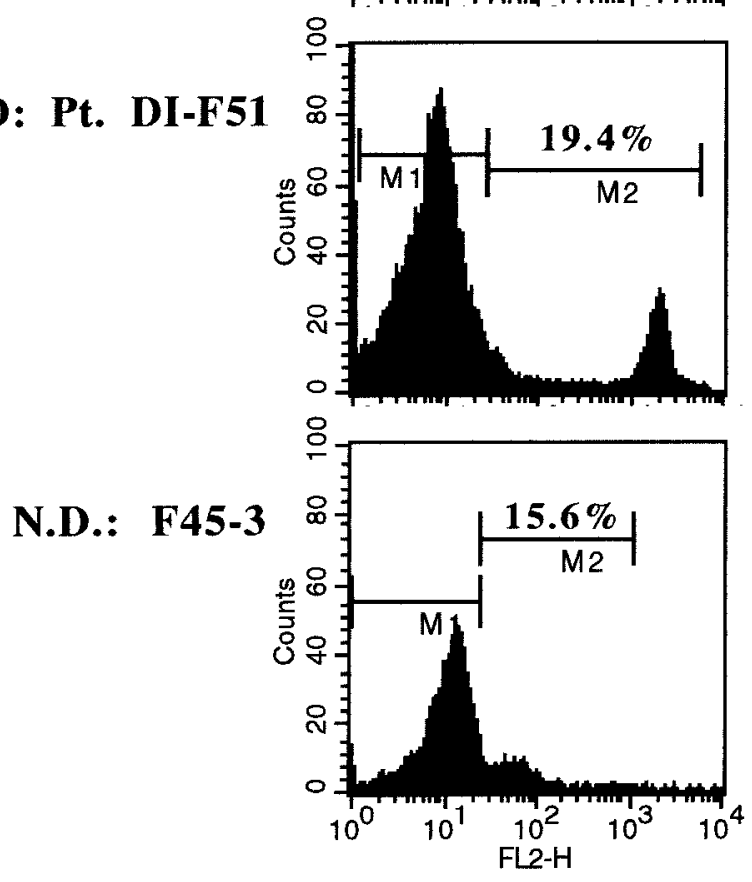

After treatment
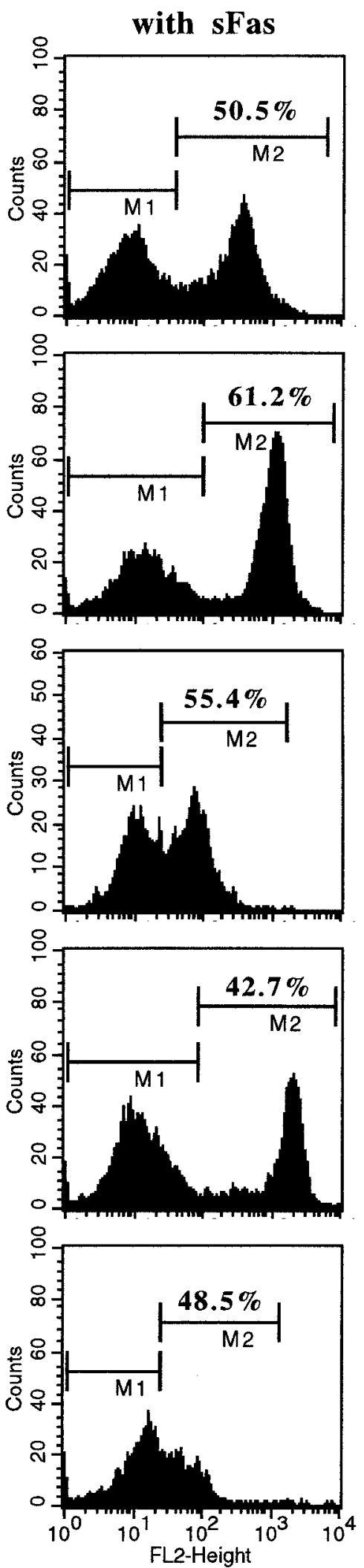

FIG. 5. Continued. 
C) showed a similar upregulation of cell death. In keeping with parallel research from our laboratory, ${ }^{27}$ recombinant Fas induced significant suppression of the proliferation rate and enhanced the expression of caspase 8 , in particular in cells from patients with minor defects of apoptosis (group D), as well as in cells from control uninfected donors. Taken together, these results suggest that sFas serum elevations may distinguish patients with severely progressing lymphopenia and that the shedding of Fas in concomitance with the dramatic activation of apoptosis may imply a molecular mechanism devoted to both spreading and amplification of the death execution program.

Antibodies to the extracellular portion of Fas may contribute to $\mathrm{T}$ cell depletion in vivo since they exert a proapoptotic effect in vitro in $\mathrm{T}$ cell lines. However, these molecules are detectable in a minority of infected subjects, independent of their clinical stage, and are thought to be elicited by an immunogenic domain of Fas located within its first consensus sequence, which is also shared by HIV-1 gp $120 .{ }^{25}$ In the present study we recorded their incidence in as few as $13 \%$ of subjects at different clinical stages, including severely lymphopenic patients with AIDS. In line with previous observations, ${ }^{5,8}$ we also detected a remarkable disposition of peripheral $\mathrm{T}$ cells to undergo apoptosis in vitro as a putative effect of their chronic stimulation in vivo by high levels of anti-Fas. In addition, the predominant expression of Fas by $\mathrm{CD}^{+}{ }^{+}$cells from these patients reflected the persistent cellular activation ${ }^{2,23}$ leading to overexpression of the receptor as the disease progresses. ${ }^{4,5}$ By contrast, the slightly reduced expression of Fas by cells from patients included in groups $\mathrm{B}$ and $\mathrm{C}$ was interpreted as possibly due to its release by dying cells.

A striking point raised by this work was the observation of increased parameters of cell death and severe disease progression in patients showing high serum titers of sFas. Our ELISA showed a relatively low incidence of sFas serum elevations in the entire HIV-1 population (26 of 227) as compared with data from other investigators. ${ }^{1,12}$ However, since our significance limit was obtained by adding 3 SD to the general mean, we assumed that our assay was highly selective for positive sera. In most subjects we observed an extreme lymphopenia consistently associated with dramatic apoptosis in vitro. These patterns of accelerated disease activity were not predominant in HIV-1-infected patients from group D, thus supporting the hypothesis of their potential relation with the amounts of circulating sFas. Both groups B and C expressed the highest incidence of AIDS as well as critical detection of apoptosis in fresh $\mathrm{T}$ cells in vitro, an event that had already occurred in vivo according to their high content of caspase products. Again, fresh $\mathrm{CD}^{+}{ }^{+}$cells from double-negativepatients showed significantly lower values of caspase 3 activation and CK18 fragment detection, whereas their moderate expression in patients from group A was presumably related to the anti-Fas chronic stimulation. The CK18 caspase product includes a 10-residue epitope exposed after caspase 3 treatment ${ }^{29}$ as a specific domain (M30) that also occurs in nonepithelial cells, such as several neuroblastoma cell lines. ${ }^{31}$

A number of studies from independent groups of investigators have emphasized the occurrence of serum elevations of soluble apoptogen receptors in HIV-1 disease, in particular both type I and II tumor necrosis factor (TNF) receptors ${ }^{17,18}$ and sFas, $, 11,12$ which are apparently independent markers of dis- ease progression. However, their maximal variations of titers have not been uniformly detected in early or advanced infection, nor has a correlation of sFas levels with clinical evolution been observed. The independence of sFas from a definite clinical stage was also evident in our study, since patients from both groups $\mathrm{B}$ and $\mathrm{C}$ showed heterogeneous disease conditions, although a prevalence of severe lymphopenia occurred.

A major issue of our observation implies the functional role of sFas, which could be involved in modulating the response of activated cells in a manner similar to that of other soluble receptors. These molecules, including receptors to interleukins, granulocyte-macrophage growth factors, ${ }^{32}$ TNFs, ${ }^{15,18}$ and HLA antigens, ${ }^{13,14}$ have been associated with the persistent activation of the immune system in many conditions. In particular, the soluble HLA molecules that occur in HIV-1 infection ${ }^{33}$ deliver an apoptotic signal in alloreactive $\mathrm{CD} 8^{+} \mathrm{T}$ cells by autocrine activation of Fas induced by upregulation of FasL. ${ }^{19}$ In our study serum sFas was found to induce in vivo caspase activation by the expression of caspase 3 , and both PARP and CK18, in subjects from both groups B and C, who showed constitutively increased apoptosis although they were not severely lymphopenic. Furthermore, the recombinant sFas highly suppressed in vitro the $\mathrm{CD}^{+}$cells of group D patients and controls, probably because these cells were not prestimulated in vivo by the soluble receptor. Since this effect was not inducible in cells functionally exhausted and advanced in their death program as in most patients with progressed disease, we reasoned that sFas could exert an antiproliferative and/or a proapoptotic effect in resting $\mathrm{T}$ cells. The prevalence of either severe lymphopenia or AIDS in group B and C patients supported our assumption.

Early investigations ${ }^{20,21}$ of soluble Fas variants secreted by activated $\mathrm{T}$ cells postulated their role as apoptosis blockers because of their capacity in vitro to disable FasL of cytotoxic cells. Although this in vitro effect has been confirmed by other studies, ${ }^{34}$ the role of sFas has not been properly evaluated in FasLnegative target cells. Therefore, HIV-1-infected patients with undetectable levels of sFas were primarily considered as having a major extent of apoptosis induced by the defect of these apoptosis blockers. ${ }^{18}$ The present study indicates that the increase in sFas levels parallels and perhaps amplifies the extent of apoptosis in vivo.

By using a number of both Fas-positive and Fas-negative T cell lines, we have demonstrated that the soluble isoform of Fas prompts apoptosis in Fas-positive cells through the Fas pathway $^{27}$ : this experimental in vitro model showed indeed a direct proapoptogen effect of sFas on cultured cells. The present work emphasizes that this in vivo mechanism aggravates the abnormal apoptosis in HIV-1 infection. In addition, it also supports data demonstrating that soluble variants of Fas induce self-association of a FasL-independent extracellular death domain of Fas leading to the receptor homooligomerization and activation of downstream caspases. ${ }^{35}$ In addition, the specific activation of both TNF- $\alpha$ receptor and Fas by short peptides homologous to their functional domains seems to act through a mirror molecular stimulation mechanism. ${ }^{36,37}$ Although we did not detect FADD, namely the Fas-associated death domain, by immunoblotting as specific product of Fas oligomerization in sFas-treated cells, the increase in the expression of caspase 8 suggested the activation of this mechanism. Since in our work the majority of patients 
with maximal $\mathrm{T}$ cell apoptosis showed the greatest serum elevations of the soluble receptor, a similar molecular mechanism could occur in vivo and further contribute to cell depletion.

\section{ACKNOWLEDGMENTS}

This work was supported by the National AIDS Research Project of the Italian Ministry of Health, ISS, Rome (1999, grant $40 \mathrm{~B} / 33$ ). The authors are grateful to V. Iacovizzi for secretarial assistance.

\section{REFERENCES}

1. Gougeon ML, Laurent-Crawford AG, Hovanessian AG, and Montagnier L: Direct and indirect mechanisms mediating apoptosis during HIV infection: Contribution to in vivo CD4 T cell depletion. Semin Immunol 1993;5:187-194.

2. Groux H, Torpier G, Monte D, Mouton V, Capron A, and Ameisen JC: Activation-induced death by apoptosis in $\mathrm{CD} 4^{+} \mathrm{T}$ cells from human immunodeficiency virus-infected asymptomatic individuals. J Exp Med 1992;175:331-340.

3. Alderson MR, Armitage RJ, Maraskovsky E, et al.: Fas transduces activation signals in normal human $\mathrm{T}$ lymphocytes. J Exp Med 1993; 178:2231-2241.

4. Katsikis PD, Wunderlich ES, Smith CA, Herzenberg LA, and Herzenberg LA: Fas antigen stimulation induces marked apoptosis of $\mathrm{T}$ lymphocytes in human immunodeficiency virus-infected individuals. J Exp Med 1995;181:2029-2036.

5. Silvestris F, Cafforio P, Frassanito MA, et al:: Overexpression of Fas antigen on T cells in advanced HIV-1 infection: Differential ligation constantly induces apoptosis. AIDS 1996;10:131-141.

6. Dhein J, Walczac H, Baumler C. Debatin K-M, and Krammer PH: Autocrine T cell suicide mediated by APO-1 (Fas/CD95). Nature 1995;373:444-448.

7. Silvestris F, Camarda G, Cafforio P, and Dammacco F: Upregulation of Fas ligand secretion in non lymphopenic stages of HIV-1 infection. AIDS 1998;12:1103-1104.

8. Silvestris F, Nagata S, Cafforio P, Silvestris N, and Dammacco F: Cross-linking of Fas by antibodies to a peculiar domain of gp120 V3 loop can enhance T cell apoptosis in HIV-1 infected patients. J Exp Med 1996;184:2287-2300.

9. Stricker K, Knipping E, Böhler T, Benner A, Krammer PH, and Debatin K-M: Anti-CD95 (APO-1/Fas) autoantibodies and T cell depletion in human immunodeficiency virus type 1 (HIV-1)-infected children. Cell Death Differ 1998;5:222-230.

10. Alnemri ES, Livingstone DJ, Nicholson DW, et al.: Human ICE/CED-3 protease nomenclature. Cell 1996;87:171-175.

11. Fadeel B, Samuelsson A, Hachiya T, Brostrom C, and Chiodi F: Elevated serum levels of soluble Fas/APO-I in human immunodeficiency virus-infected individuals. Blood 1996;89:4727-4730.

12. Hosaka N, Oyazu N, Kaplan MH, Yagita H, and Pahwa S: Membrane and soluble forms of Fas (CD95) and Fas ligand in peripheral blood mononuclear cells and in plasma from human immunodeficiency virus-infected persons. J Infect Dis 1998;178:10301039.

13. Liem LM, Koelman CA, Doxiadis II, van Houwelingen JC, and Goulmy E: Elevated serum HLA class I levels coincide with acute and chronic graft-versus-host disease. Bone Marrow Transplant 1997;20:227-234.

14. Zavazava N, Westphal E, and Muller-Rucholtz W: Characterization of soluble HLA molecules and quantitative HLA differences in serum of healthy individuals. J Immunogenet 1991;17:387-394.
15. Heilig B, Fiehn C, Brockhaus M, Gallati H, Pezzuto A, and Hunstein W: Evaluation of soluble tumor necrosis factor (TNF) receptors and TNF receptor antibodies in patients with systemic lupus erythematosus, progressive systemic sclerosis, and mixed connective tissue disease. J Clin Immunol 1993;13:321-328.

16. Nozawa K, Kayagaki N, Tokano Y, Yagita H, Okumura K, and Hasimoto H: Soluble Fas (APO-I, CD95) and soluble Fas ligand in rheumatic diseases. Arthritis Rheum 1997;40:1126-1129.

17. Godfried MH, Van der Poll T, Mulder R, et al:: Soluble receptors for tumor necrosis factor as predictors of progression to AIDS in asymptomatic human immunodeficiency virus type 1 infection. J Infect Dis 1994;169:739-745.

18. Medrano FJ, Leal M, Arienti $\mathrm{D}$, et al.: Tumor necrosis factor $\beta$ and soluble APO-1/Fas independently predict progression to AIDS in HIV seropositive patients. AIDS Res Hum Retroviruses 1998; 14:835-843.

19. Zavazava N and Krönke M. Soluble HLA class I molecules induce apoptosis in alloreactive cytotoxic T lymphocytes. Nat Med 1996; 2:1005-1010.

20. Papoff G, Cascino I, Eramo A, Starace G, Lynch DH, and Ruberti G: An N-terminal domain shared by Fas/APO-I (CD95) soluble variants prevents cell death in vitro. J Immunol 1996;156: 4622-4630.

21. Cheng J, Zhou T, Liu C, et al.: Protection from Fas-mediated apoptosis by a soluble form of the Fas molecule. Science 1994;263: 1759-1762.

22. Knipping E, Debatin K-M, Stricker K, Heilig B, Eder A, and Krammer PH: Identification of soluble APO-1 in supernatants of human $\mathrm{B}$ and $\mathrm{T}$ cell lines and increased serum levels in $\mathrm{B}$ and $\mathrm{T}$ cell leukemias. Blood 1995;85:1562-1569.

23. Bäumler C, Herr I, Böhler T, et al.: Activation of the CD95 (APO$1 /$ Fas) system in $T$ cells from human immunodeficiency virus type1 infected children. Blood 1996;88:1741-1746.

24. Center for Disease Control and Prevention: 1992 Revised classification system for HIV infection and expanded surveillance case definition for AIDS among adolescent and adults. MMWR 1992; 41/RR-17:1-19.

25. Silvestris F, Cocco T, Cafforio P, Calvani N, and Dammacco F: Immunogenicity of an eight aminoacid domain shared by Fas (CD95/Apo-I) and HIV-1 gp120. I. Structural and antigenic analysis. Mol Med 2000;6:494-508.

26. Koester SK, Roth P, Mikulka WR, Schlossman SF, Zhang C, and Bolton WE: Monitoring early cellular responses in apoptosis is aided by the mitochondrial membrane protein-specific monoclonal antibody APO2.7. Cytometry 1997;29:306-312.

27. Silvestris F, Cafforio P, Tucci M, Del Prete A, and Dammacco F: VEINCTR-N, an immunogenic epitope of Fas (CD95/Apo-I), and soluble Fas enhance T cell apoptosis in vitro. II. Functional analysis and possible implications in HIV-1 disease. Mol Med 2000; 6:509-526.

28. Silvestris F, Tucci M, Cafforio P, and Dammacco F: Fas-L upregulation by highly malignant myeloma plasma cells: Role in the pathogenesis of anemia and disease progression. Blood 2001;97: 1155-1164.

29. Leers MP, Kolgen W, Bjorklund V, et al.: Immunocytochemical detection and mapping of a cytokeratin 18 neo-epitope exposed during early apoptosis. J Pathol 1999;187:567-572.

30. Silvestris F, Cafforio P, Romito A, and Dammacco F: Molecular specificities of $\mathrm{CD}^{+}{ }^{+} \mathrm{T}$ cell reactive $\mathrm{IgG}$ and $\mathrm{IgM}$ in human immunodeficiency virus (HIV-1) infection. Clin Immunol Immunopathol 1994;70:40-46.

31. Van Engeland M, Knijpers HJ, Ramaekers FC, Reutelingsperger $\mathrm{CP}$, and Schutte B: Plasma membrane alterations and cytoskeletal changes in apoptosis. Exp Cell Res 1997;235:421-430.

32. Asworth A and Kraft A: Cloning of a potential soluble receptor for human GM-CSF. Nucleic Acids Res 1990;18:7178-7183. 
33. Filaci G, Contini $\mathrm{P}$, Brenci $\mathrm{S}$, et al.: Increased serum concentration of soluble HLA-DR antigens in HIV infection and following transplantation. Tissue Antigens 1995;46:117-123.

34. Suda T, Hashimoto H, Tanaka M, Ochi T, and Nagata S: Membrane Fas ligand kills human peripheral blood $\mathrm{T}$ lymphocytes, and soluble Fas blocks the killing. J Exp Med 1997;186: 2045-2050.

35. Papoff G, Hauser P, Eramo A, et al.: Identification and characterization of a ligand-independent oligomerization domain in the extracellular region of the CD95 death domain receptor. J Biol Chem 1999;274:38241-38250

36. Boldin MP, Varfolomeev EE, Pancer Z, Mett IL, Camonis JH, and Wallach D: A novel protein that interacts with the death domain of Fas/ApoI contains a sequence motif related to the death domain. J Biol Chem 1995;270:7795-7798.
37. Boldin MP, Mett IL, Varfolomeev EE, et al.: Self-association of the "death domain" of the p55 tumor necrosis factor (TNF) receptor and Fas/ApoI prompts signaling for TNF and Fas/ApoI effects. J Biol Chem 1995;270:387-391.

Address reprint requests to: Franco Silvestris

DIMO, University of Bari Section of Internal Medicine P.za Giulio Cesare, 11 70124, Bari, Italy

E-mail: f.silvestris@dimo.uniba.it 